\title{
Association between radical cystectomy prophylactic antimicrobial regimen and postoperative infection
}

\author{
James Paul Joseph Ross, MD'; Rodney H. Breau, MD',2,3; Humberto Vigil, MD'; Duane R. Hickling, MD',2; \\ Jonathan B. Angel, MD2,4; Ranjeeta Mallick, MD'; Ilias Cagiannos, MD'; Christopher Morash, MD', \\ Luke T. Lavallée, $M D^{1,2,3}$
}

'Division of Urology, Department of Surgery, University of Ottawa, Ottawa, ON, Canada; ${ }^{2 T h e}$ Ottawa Hospital Research Institute, Ottawa, ON, Canada; ${ }^{3}$ School of Epidemiology and Public Health, Faculty of Medicine, University of Ottawa, Ottawa, ON, Canada; ${ }^{4}$ Division of Infectious Diseases, Department of Medicine, University of Ottawa, Ottawa, ON, Canada

Cite as: Ross JPJ, Breau RH, Vigil H, et al. Association between radical cystectomy prophylactic antimicrobial regimen and postoperative infection. Can Urol Assoc J 2021;15(12):E644-51. http://dx.doi.org/10.5489/cuaj.7158

Published online June 22, 2021

\section{Abstract}

Introduction: Infections are common after radical cystectomy. The objective of this study was to determine the association between antimicrobial prophylactic regimen and infection after radical cystectomy. Methods: A retrospective cohort study was performed on patients who underwent radical cystectomy at one tertiary Canadian center between January 2016 and April 2020. Patients received antimicrobial prophylaxis based on surgeon preference (cefazolin/metronidazole or ampicillin/ciprofloxacin/metronidazole, or other). A univariable and multivariable logistic regression model was created to determine the association between antimicrobial regimen and postoperative infection within 30 days. The association between patient demographic factors, as well as preoperative and intraoperative variables and infection, was also determined. Infection characteristics, including type, timing, and antimicrobial susceptibilities were reported.

Results: One hundred and sixty-five patients were included. Mean age was 69.8 years, 121 (73.3\%) were male, and $72(43.6 \%)$ received orthotopic neobladder diversion. Ninety-six patients $(58 \%)$ received cefazolin/metronidazole prophylaxis, $50(30 \%)$ received ampicillin/ciprofloxacin/metronidazole, and 19 (11.5\%) received another regimen. Fifty-four patients (32.7\%) developed a postoperative infection (surgical site infection or urinary tract infection). Surgical site infection occurred in 35 patients $(21.2 \%)$ and urinary tract infection occurred in 34 (21.0\%). There was no association between antimicrobial regimen and incidence of postoperative infection (surgical site infection or urinary tract infection, relative risk 0.99 , 95\% confidence interval 0.50-1.99).

Conclusions: The overall incidence of infection was $32.7 \%$ following radical cystectomy. The preoperative prophylactic antibiotic regimen used was not associated with incidence of postoperative infection.

\section{Introduction}

Approximately $60 \%$ of radical cystectomy patients will develop a postoperative complication. ${ }^{1-4}$ Infection, specifically urinary tract infection (UTI), surgical site infection (SSI), and postoperative Clostridium difficile (C. diff) infection, are among the most common, with reported rates of SSI up to $33 \%$, UTI up to $36 \%$, and C. diff up to $12 \% .^{1,2,4-9}$ The incidence of infection remains high in recent series and has been shown to negatively impact quality of life, prolong hospital stay, and increase healthcare-related costs..$^{8,10-13}$

Despite a high incidence of infection, there are few interventions proven to reduce rates after radical cystectomy. Previously, we reported a simple intraoperative intervention bundle with a wound barrier that reduced SSIs by $80 \% .^{14}$ Preoperative prophylactic antimicrobials have been shown to reduce postoperative infection in surgery, however, cystectomy-specific data are lacking. The American Urological Association (AUA) and European Association of Urology (EAU) recommend that patients undergoing radical cystectomy and diversion receive antimicrobial prophylaxis based on colorectal surgery data. ${ }^{15-18}$ Further study is needed to characterize infections in patients undergoing radical cystectomy and determine the best methods of prevention.

The primary objective of this study was to determine the association between prophylactic antimicrobial regimens and infection (SSI/UTI) after cystectomy. Secondary objectives were to: characterize the incidence and timing of SSIs and UTI after cystectomy within 30 days, identify common causative organisms and antimicrobial susceptibilities, determine the incidence of postoperative $C$. diff infection, and identify patient and surgery risk factors for SSI/UTI. These data will be used to improve infection prevention protocols and to identify high-risk patients and settings requiring further study. 


\section{Methods}

\section{Study overview}

A retrospective cohort study was performed on all patients who underwent radical cystectomy by one of four uro-oncologists at The Ottawa Hospital between January 1, 2016 , and April 1, 2020. Patients were excluded if they received cystectomy by a non-oncologic surgeon, if they did not have a primary diagnosis of bladder cancer (e.g., exenteration for rectal cancer), or if they underwent simple or partial cystectomy. Institutional ethics approval was obtained.

\section{Perioperative infection prevention}

All patients received antimicrobial prophylaxis the day of the surgery immediately prior to anesthesia and skin incision. Most patients received cefazolin/metronidazole or ampicillin/metronidazole/ciprofloxacin. The antimicrobial regimen was chosen at the surgeon's discretion and was based on local practice antibiograms and guidelines for patients undergoing surgery involving the intestine. Some patients received another regimen based on patient-specific factors (e.g., allergies to a specific antimicrobial, preoperative urine culture result, or prior history of infection). Antimicrobials were re-dosed during the operation as per institutional best practices. Antimicrobials were routinely continued for 24 hours postoperatively. All patient's received preoperative urine cultures the week prior to surgery. Patients with positive preoperative urine cultures may have received additional antimicrobials preoperatively based on culture sensitivities at the surgeon's discretion. Stent removal following ileal conduit creation was usually performed on postoperative day 7-14; catheter and stent removal following neobladder urinary diversion was performed approximately 21 days postoperatively. The timing of stent and catheter removal, and use of antimicrobials before/after these procedures, was at the discretion of the treating surgeon and based on the patient's clinical status.

An intraoperative SSI prevention bundle was introduced for all patients undergoing radical cystectomy on or after March 1, 2018. The bundle was designed to reduce incisional SSIs and consisted of barrier wound protection (Alexis retractor), gown and glove change prior to fascial closure, use of a separate sterile closing tray, washing the wound with a proviodine/saline mix following fascial closure, and application of an antimicrobial impregnated dressing. The impact of the SSI prevention bundle was previously reported. ${ }^{14}$ Use of the SSI prevention bundle did not impact the antimicrobial regimen choice and it was included in the multivariable analysis to adjust for possible confounding.

\section{Study outcomes}

The primary outcome was the association between prophylactic antimicrobial regimens (cefazolin/metronidazole vs. ampicillin/ciprofloxacin/metronidazole vs. other) and postoperative infection (SSI or UTI) within 30 days of surgery. All SSIs or UTIs within 30 days of surgery were included in the analysis. Secondary outcomes were to characterize the incidence and timing of SSIs and UTI, identify common causative organisms and antimicrobial susceptibilities, determine the incidence of postoperative $C$. diff infection, and identify patient and surgical risk factors for SSI/UTI.

The Center for Disease Control definitions were used for SSI and UTI. ${ }^{19}$ As per the CDC, SSIs are separated according to tissue layers involved and included superficial infections involving the skin and subcutaneous tissue, deep infections involving the facial and muscle layers, and organ space infections involving any space deep to the fascia that was disrupted during the operation. Patients meeting the criteria for infection for any tissue layer were included as an SSI event. UTI was defined as a positive urine culture in the presence of associated symptoms, including fatigue, pressure, abdominal pain, foul-smelling urine, cloudy urine, and/or fever. Sepsis related to the underlying infection was identified using the Systematic Inflammatory Response Syndrome (SIRS) criteria. ${ }^{20}$ Time to infection was defined as the time from operation to positive culture result or clinical diagnosis of infection for SSI if no culture was available.

\section{Data collection}

A prospective cystectomy database is maintained at The Ottawa Hospital. The cystectomy database includes patient, tumor, and operative characteristics. A single reviewer (JR) reviewed all medical records retrospectively for antimicrobial- and infection-related variables and outcomes. Patient variables collected included sex, age, body mass index (BMI), smoking status, diagnosis of diabetes, and whether patient received neoadjuvant chemotherapy. Operative variables collected included operation date, operation time, preoperative prophylactic antimicrobial regimen (cefazolin/ metronidazole vs. ampicillin/ciprofloxacin/metronidazole vs. other), estimated blood loss (EBL), need for intraoperative blood transfusion, type of urinary diversion performed (ileal conduit vs. orthotopic neobladder vs. other), and pathological stage. Postoperative variables collected included SSI, UTI, and C. diff infection within 30 days of operation, culture results, antimicrobial susceptibilities of cultured bacteria, time to infection, re-admission for infection, whether UTI was associated with catheter or stent removal, and incidence of sepsis from infection. 


\section{Analysis}

Continuous and categorical baseline patient variables were organized by antimicrobial prophylaxis regimen used and were compared using t-tests and Chi-squared tests, respectively. A composite outcome of infection (either SSI or UTI) was used for the primary analysis. Univariable and multivariable log binomial regression models were used to determine the association between antimicrobial prophylaxis regimens on postoperative infection. Models also determined the associations between other patient and operative factors with the primary outcome. The surgeon was included in the multivariable analysis as a cluster variable to adjust for random effect of surgeon-specific factors. For each postoperative infection (SSI or UTI), urine, wound, and blood cultures were reviewed. For each positive culture, all microorganisms isolated were identified. For bacteria, antimicrobial susceptibility was recorded. Antimicrobial susceptibility results were limited to what was reported by the local microbiology laboratory. The percentage of bacteria that were resistant to each antimicrobial type tested was reported. Statistical significance was defined as $p<0.05$. All analysis was performed using SAS software, version 9.4 (SAS Institute Inc.).

\section{Results}

One hundred and sixty-five patients met the inclusion criteria. The mean patient age at time of cystectomy was 69.8 years (standard deviation [SD] 10.2). One-hundred and twenty-one $(73.3 \%)$ were male and $77(46.7 \%)$ received neoadjuvant chemotherapy. Eighty-eight (53.3\%) received ileal conduit diversion, 72 (43.6\%) underwent orthotopic neobladder diversion, and five patients $(3.0 \%)$ underwent an alternative diversion, which included colon conduit in three patients $(1.8 \%)$ and no diversion in two patients $(1.6 \%)$ who underwent concomitant removal of their entire urinary tract. For antimicrobial prophylaxis, 96 (58\%) received cefazolin/ metronidazole, 50 (30\%) received ampicillin/metronidazole/ ciprofloxacin, and 19 patients $(12 \%)$ received an alternative regimen. Further information on baseline characteristics between patient groups can be seen in Table 1 .

In total, 35 patients $(35 / 165 ; 21.2 \%)$ developed an SSI and 34 patients $(34 / 165 ; 21.0 \%)$ developed a UTI for a total of 69 individual infections. Fifty-four patients $(54 / 165 ; 32.7 \%)$ developed either SSI or UTI, with 15 patients (15/165; 9.1\%) having both (Table 2). The median time to infection for both SSI and UTI was 12 days. There was a peak in SSIs from postoperative days 7-9 and 19-21, and in UTIs from postoperative days 7-9 and 16-18 (Fig. 1). Four patients developed UTI following either catheter or stent removal $(4 / 165 ; 2.4 \%)$. Forty-three infections $(43 / 69 ; 62.3 \%)$ occurred during the index admission after surgery, 22 infections occurred after discharge and required re-admission to hospital (22/69;
$31.9 \%)$, and 32 infections were associated with sepsis $(32 / 69 ; 46.4 \%)$. The most common causative organism was Klebsiella sp. for SSI $(11 / 35 ; 31.4 \%)$ and E. coli for UTI (9/34; 26.5\%) (Fig. 2). Thirteen infections (13/69; 18.8\%) had $\geq 2$ organisms cultured. Ten infections were fungal (10/69; $14.5 \%)$ : five $(5 / 96,5.2 \%)$ in the cefazolin/metronidazole group and five in the ampicillin/ciprofloxacin/metronidazole group (5/50, 10\%) (relative risk [RR] 0.56, 95\% confidence interval $[\mathrm{Cl}]<0.001,0.707, \mathrm{p}=0.10)$.

In multivariable analyses, prophylactic antimicrobial regimen was not associated with overall infection (ampicillin/ ciprofloxacin/metronidazol vs. cefazolin/metronidazol; RR 0.99, 95\% Cl 0.50-1.99) (Table 2). There was a non-statistically significant increased risk of infection for patients with higher BMI, patients with higher American Society of Anesthesiologists (ASA) score, those who received an intraoperative transfusion, and those who received a neobladder urinary diversion (Fig. 3). Separate multivariable analyses were performed for the SSI outcome alone and the UTI outcome alone. This was done because it is possible that opposing directions of association for SSI and UTI could confound a clinically important association. Neobladder urinary diversion trended towards a decreased risk of SSI (RR $0.72,95 \% \mathrm{Cl} 0.39,1.33, \mathrm{p}=0.30)$ and an increased risk of UTI (RR 1.98, 95\% Cl 0.80, 4.90, p=0.14) compared to ileal conduit. Positive preoperative urine culture trended towards a decreased risk of infection (Fig. 3).

For bacteria isolated from all positive UTI or SSI cultures, resistance to antibiotics were frequently noted. Of the bacteria cultured, resistance to a combination of ampicillin/ ciprofloxacin/metronidazole was seen in $22 \%$ and resistance to a combination of cefazolin/metronidazole was seen in $30 \%$ (Fig. 4). Twenty-one patients $(21 / 54,38.9 \%)$ had infections resistance to $\geq 2$ antimicrobials. C. diff infection occurred in nine patients $(5.5 \%$ ) (six patients for cefazolin/metronidazole and three for ampicillin/ciprofloxacin/ metronidazole, RR 0.71, $\mathrm{Cl}<0.001,70.4, \mathrm{p}=0.59$ ) (Table 2).

\section{Discussion}

Infection is a common sources of morbidity after cystectomy. ${ }^{2,15,16}$ In this study, there was no association between the prophylactic antimicrobial regimen used and postoperative infections. Infection (SSI or UTI) occurred in $32.7 \%$ of patients, which is comparable to other institutions., ${ }^{4,21-23}$ Among patients with infection, there was a high rate of associated sepsis (48.5\%) and hospital re-admission (33.3\%). 4,7,22 Possible reasons for high infection rate include: insufficient antimicrobial coverage, duration, or timing; decreased susceptibility of bacteria to some antimicrobials; frequent presence of fungi; and a high number of patient- and procedure-specific risk factors.

This study did not identify an association between antimicrobial regimen and incidence of infection. Other studies 


\begin{tabular}{|c|c|c|c|c|c|}
\hline Variable & $\begin{array}{l}\text { Cefazolin/metronidazole } \\
n=96(58 \%)\end{array}$ & $\begin{array}{l}\text { Ampicillin/ciprofloxacin/metronidazole } \\
\qquad n=50(30 \%)\end{array}$ & $\begin{array}{c}\text { Other } \\
\mathrm{n}=19(11.5 \%)\end{array}$ & $\begin{array}{c}\text { Total } \\
\mathrm{n}=165\end{array}$ & $\mathbf{p}$ \\
\hline \multicolumn{6}{|l|}{ Sex, n (\%) } \\
\hline Male & 70 (72.9) & $38(76.0)$ & $13(68.4)$ & $121(73.3)$ & 0.81 \\
\hline Female & $26(27.1)$ & $12(24.0)$ & $6(31.6)$ & $44(26.7)$ & \\
\hline \multicolumn{6}{|l|}{ Age, $\mathrm{n}(\%)$} \\
\hline Mean (SD) & $70.1(9.5)$ & $69.6(10.9)$ & $68.7(11.6)$ & $69.8(10.2)$ & 0.85 \\
\hline$<60$ & $14(14.6)$ & $8(16.0)$ & $5(26.3)$ & 27 (16.4) & 0.76 \\
\hline 60 to $<70$ & $29(30.2)$ & $14(28.0)$ & $3(15.8)$ & 46 (27.9) & \\
\hline 70 to $<80$ & 37 (38.5) & $19(38.0)$ & $9(47.4)$ & 65 (39.4) & \\
\hline$\geq 80$ & 16 (16.7) & $9(18.0)$ & $2(10.5)$ & 27 (16.4) & \\
\hline \multicolumn{6}{|l|}{ BMI, n (\%) } \\
\hline Mean (SD) & $28.2(4.35)$ & $27.8(5.8)$ & $26.4(4.7)$ & $27.9(4.9)$ & 0.33 \\
\hline$<30$ & $67(71.3)$ & $30(60.0)$ & $16(84.2)$ & $113(69.3)$ & 0.12 \\
\hline$\geq 30$ & $27(28.7)$ & $20(40.0)$ & $3(15.8)$ & $50(30.7)$ & \\
\hline \multicolumn{6}{|l|}{ Diabetes n (\%) } \\
\hline Yes & $20(20.8)$ & $11(22.0)$ & $2(10.5)$ & $33(20.0)$ & 0.54 \\
\hline No & $76(79.2)$ & $39(78.0)$ & 17 (89.5) & $132(80.0)$ & \\
\hline \multicolumn{6}{|l|}{ Smoking } \\
\hline Yes & 75 (78.1) & $40(80.0)$ & 15 (78.9) & $130(78.8)$ & 0.97 \\
\hline No & 21 (21.9) & $10(20.0)$ & $4(21.1)$ & $35(21.2)$ & \\
\hline \multicolumn{6}{|c|}{ Neoadjuvant chemo, n (\%) } \\
\hline Yes & $47(49.0)$ & $25(50.0)$ & $5(26.3)$ & $77(46.7)$ & 0.17 \\
\hline No & $49(51.0)$ & $25(50.0)$ & $14(73.7)$ & $88(53.3)$ & \\
\hline \multicolumn{6}{|c|}{ ASA class, n (\%) } \\
\hline II & $6(6.2)$ & $3(6.0)$ & $1(5.3)$ & $10(6.1)$ & 0.25 \\
\hline III & $86(89.6)$ & $46(92.0)$ & $15(78.9)$ & $147(89.1)$ & \\
\hline IV & $4(4.2)$ & $1(2.0)$ & $3(15.8)$ & $8(4.8)$ & \\
\hline \multicolumn{6}{|c|}{ Diversion, n (\%) } \\
\hline Ileal conduit & $44(45.8)$ & $31(62.0)$ & $13(68.4)$ & $88(53.3)$ & 0.020 \\
\hline Neobladder & $49(51.0)$ & $19(38.0)$ & $4(21.1)$ & $72(43.6)$ & \\
\hline Other & $3(3.1)$ & $0(0.0)$ & $2(10.5)$ & $5(3.0)$ & \\
\hline \multicolumn{6}{|c|}{ Operative time, n (\%) } \\
\hline Med (IQR) & $471(403,536)$ & $419(377,466)$ & $463(377,570)$ & $454(396,519)$ & \\
\hline$<420$ mins & $29(30.2)$ & $25(50.0)$ & $6(31.6)$ & $60(36.4)$ & 0.0055 \\
\hline$\geq 420$ mins & 67 (69.8) & $25(50.0)$ & $13(68.4)$ & $105(63.6)$ & 0.056 \\
\hline \multicolumn{6}{|l|}{$\mathrm{EBL}, \mathrm{n}(\%)$} \\
\hline Med (IQR) & $1000(650,1500)$ & $800(500,1120)$ & $900(775,1200)$ & $950(600,1300)$ & 0.036 \\
\hline$<1 \mathrm{~L}$ & $43(44.8)$ & $30(60.0)$ & $10(52.6)$ & $83(50.3)$ & 0.21 \\
\hline$\geq 1 \mathrm{~L}$ & $53(55.2)$ & $20(40.0)$ & $9(47.3)$ & $82(49.7)$ & \\
\hline \multicolumn{6}{|c|}{ Transfusion, n (\%) } \\
\hline Yes & 37 (38.5) & $15(30.0)$ & $6(31.6)$ & $58(35.2)$ & 0.56 \\
\hline No & $59(35.8)$ & $35(70.0)$ & $13(68.4)$ & $107(64.8)$ & \\
\hline \multicolumn{6}{|c|}{ Final pathology } \\
\hline MIBC & $52(54.2)$ & $23(46.0)$ & $11(57.9)$ & $86(52.1)$ & 0.56 \\
\hline NMIBC & $44(26.7)$ & $27(54.0)$ & $8(42.1)$ & 79 (47.9) & \\
\hline \multicolumn{6}{|c|}{ SSI prev. bdl, ${ }^{*}$ n (\%) } \\
\hline Yes & $34(35.4)$ & $34(68.0)$ & $6(31.6)$ & $74(44.8)$ & 0.0004 \\
\hline No & $62(64.6)$ & $16(32.0)$ & $13(68.4)$ & $91(55.2)$ & \\
\hline \multicolumn{6}{|c|}{ Preop urine $\mathrm{Cx}, \mathrm{n}(\%)$} \\
\hline Positive & $25(26.0)$ & $10(21.3)$ & $7(36.8)$ & $42(25.9)$ & 0.44 \\
\hline Negative & $71(74.0)$ & $37(78.7)$ & $12(63.2)$ & $120(74.1)$ & \\
\hline
\end{tabular}

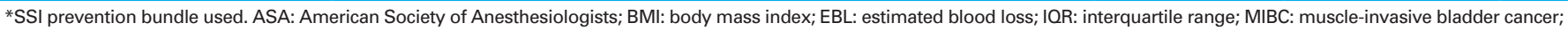
NMIBC: non-muscle-invasive bladder cancer; SD: standard deviation; SSI: surgical site infection.

have found reduced infections with broader antimicrobial coverage. One retrospective cohort study demonstrated a decreased risk of overall infection from $41 \%$ to $30 \%$ $(p=0.045)$ with use of ampicillin-sulbactam, gentamicin, and fluconazole compared to cefoxitin alone. ${ }^{21}$ Another study found for patients receiving penicillin-based prophylaxis, the 


\begin{tabular}{|c|c|c|c|c|c|}
\hline Infection & $\begin{array}{l}\text { Cefazolin/metronidazole } \\
\qquad n=96\end{array}$ & $\begin{array}{l}\text { Ampicillin/ciprofloxacin/metronidazole } \\
\qquad n=50\end{array}$ & Total $n=165$ & RR (95\% Cl) & $\mathbf{p}$ \\
\hline Overall (SSI or UTI) & $34(35.4)$ & $14(28.0)$ & $54(32.7)$ & $0.99(0.50-1.99)$ & 0.75 \\
\hline SSI & 22 (22.9) & $9(18.0)$ & $35(21.2)$ & $1.08(0.46,2.50$ & 0.86 \\
\hline UTI & $22(22.9)$ & $9(18.0)$ & $34(20.6)$ & $1.03(0.40,2.68)$ & 0.94 \\
\hline C. diff & $6(6.3)$ & $3(6.0)$ & $9(5.5)$ & $0.71(<0.001,70.4)$ & 0.59 \\
\hline
\end{tabular}

incidence of infection decreased with the addition of a betalactamase inhibitor. ${ }^{2}$ All patients in this study received broad antimicrobial regimens with both aerobic and anaerobic coverage. This may have resulted in the lack of significant difference in incidence of infection between regimens.

The AUA recommends a single dose of prophylactic cefazolin preoperatively, with no direct recommendations for extending antimicrobials beyond this time period or use of prophylaxis at the time of catheter and stent removal. Prior studies have reported mixed results regarding UTI risk around the time of catheter and stent removal. One retrospective cohort study found that $37 \%$ of all UTIs postcystectomy occurred within 24 hours of stent removal. ${ }^{24}$ Another retrospective review found a significant decrease in UTI with administration of prophylactic antimicrobials at time of ureteric stent removal post-urinary diversion. ${ }^{25}$ In contrast, two other retrospective studies did not find a benefit of extending antimicrobials beyond 24 hours. ${ }^{2,4}$ In this study, patients were given antimicrobials for 24 hours postoperatively. Routine use of antimicrobials was not mandated at the time of catheter or stent removal, but patients may have received antimicrobial prophylaxis at the discretion of their treating surgeon. In total, four patients $(4 / 165 ; 2.4 \%)$ developed UTIs associated with catheter or stent removal.

In this study, higher BMI, female sex, higher ASA score, and intraoperative transfusion were associated with increased

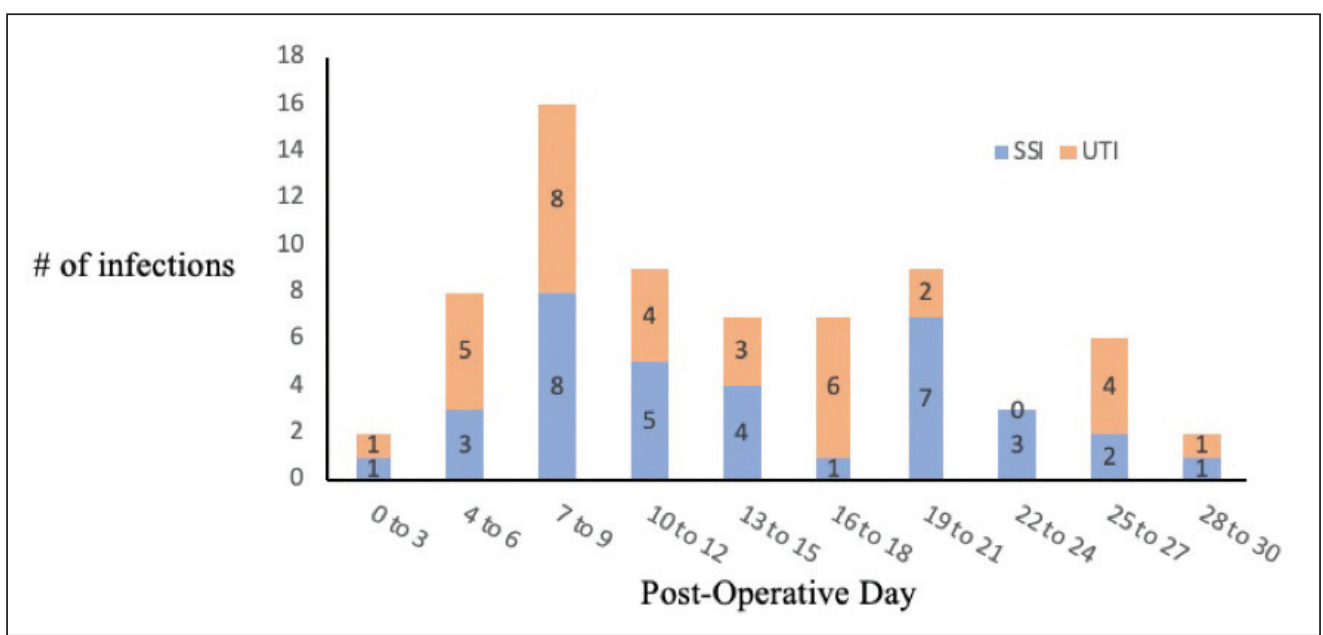

Fig. 1. Number of surgical site infections (SSI) and urinary tract infections (UTI) by postoperative day from radical cystectomy. risk of infection. Similar to other studies, patients receiving continent urinary diversion appeared to have a higher risk of UTI compared to ileal conduit. 4,24,26,27 Active infection at time of cystectomy has been associated with increased risk of postoperative infection. ${ }^{24}$ In this study, patients with a positive preoperative urine culture had less infections. We hypothesize this could be because patients with positive preoperative culture may have received targeted preoperative antimicrobials, which sterilized their urinary tract prior to surgery, making the treatment more directed and effective. The incidence of postoperative C. diff was low in this study, at $5.5 \%$, and prophylactic antimicrobial regimen was not associated with increased risk of $C$. diff.

E. coli was the most common cause of UTI $(29.0 \%$ of UTIs), and Klebsiella sp. was the most common cause of SSI (31.4\% of SSIs). Other studies have identified E. coli and Enterococcus as a common cause of UTI and Enterococcus, Klebsiella, and coagulase-negative Staphylococcus as common causes of SSI in this population. ${ }^{1,4-7,21,28,29}$ Fungal infections have also been reported in up to $23 \%$ of cystectomy patients. ${ }^{1,7,21}$ Candida was isolated in $15 \%$ of SSI or UTI cultures in this study, with no difference noted between prophylaxis regimens given.

Frequently, cultured bacteria were not susceptible to some of the antimicrobials used; for example, $22 \%$ of cultures isolated a bacteria that was resistant to the ampicillin/ciprofloxacin/metronidazole regimen, and $30 \%$ of cultures identified a bacteria resistant to the cefazolin/metronidazole regimen. While some of the susceptibility/resistance patterns identified may be the result of antimicrobial pressures from the prophylaxis, these resistance patterns illustrate the challenge posed by patients with frequent antimicrobial exposures. Other studies have also demonstrated high incidence of resistant organisms, including vancomycin-resistant enterococcus in up to $12.8 \%$, and methicillin-resistant staphylococcus in 


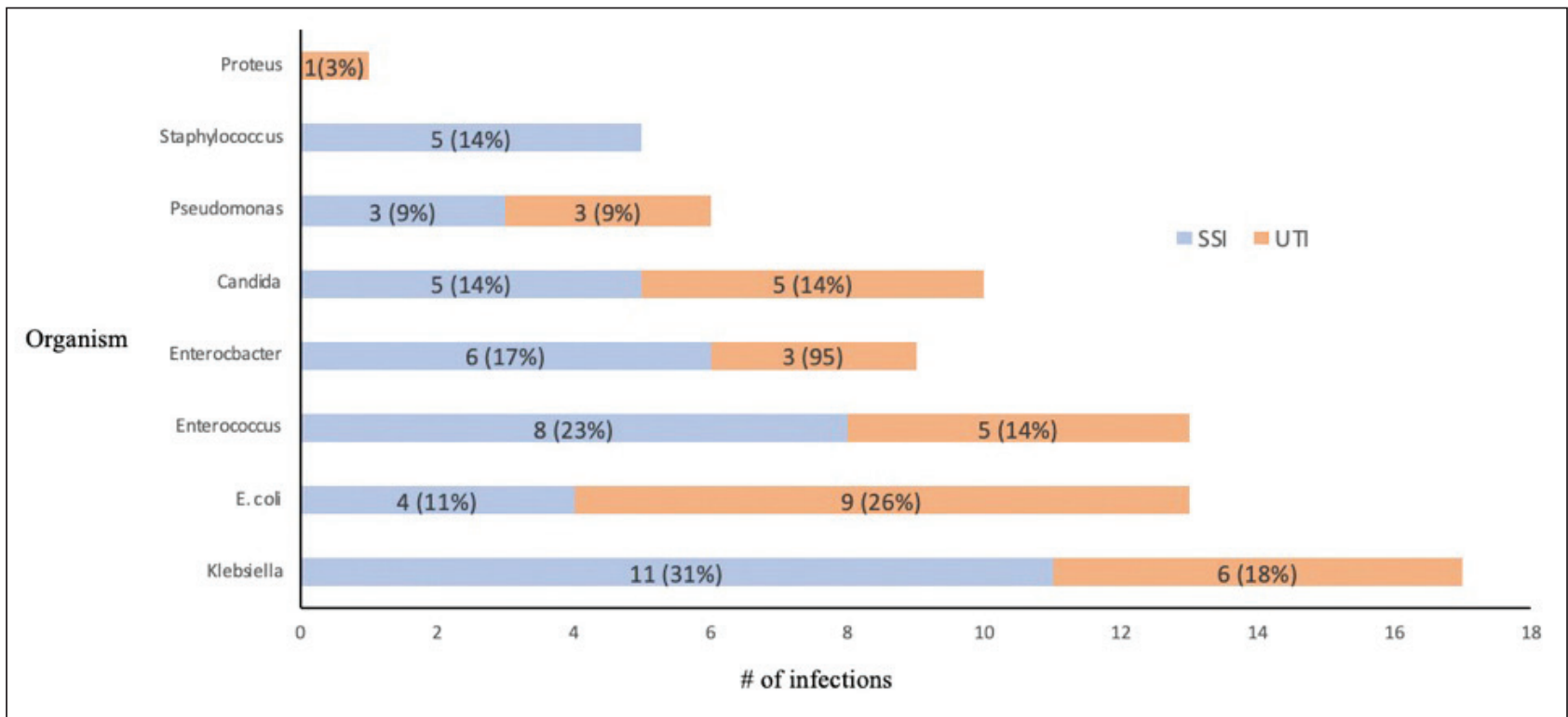

Fig. 2. Frequency of organisms isolated from postoperative culture for surgical site infection (SSI) and urinary tract infection (UTI).

up to $63.6 \% .{ }^{1,24}$ This high level of antimicrobial resistance highlights the importance of adherence to institution-specific antibiograms when making decisions regarding antimicrobial coverage. Furthermore, promotion of antimicrobial stewardship and development of non-antimicrobial solutions to reduce infection is required. These approaches may include identification of high-risk populations and modifying procedure and patient risk factors perioperatively when possible.

The proportion of bacteria that were resistant (not susceptible) to specific antimicrobials was reported. These results are meant to provide insight into interventions that may reduce infections in this population. It is not meant to represent a local antibiogram, because resistance to antimicrobials identified in this study may be a consequence of antimicrobials used during prophylaxis, and therefore may not accurately represent true susceptibilities prior to surgery. Otherwise stated, the antimicrobial prophylactic regimen may have induced resistances. While this is a limitation of the study design, results do suggest that common infective organisms, including E. coli and Klebsiella, are usually susceptible to nitrofurantoin. Other studies have also reported low resistance rates to nitrofurantoin $(21 \%)$, suggesting it

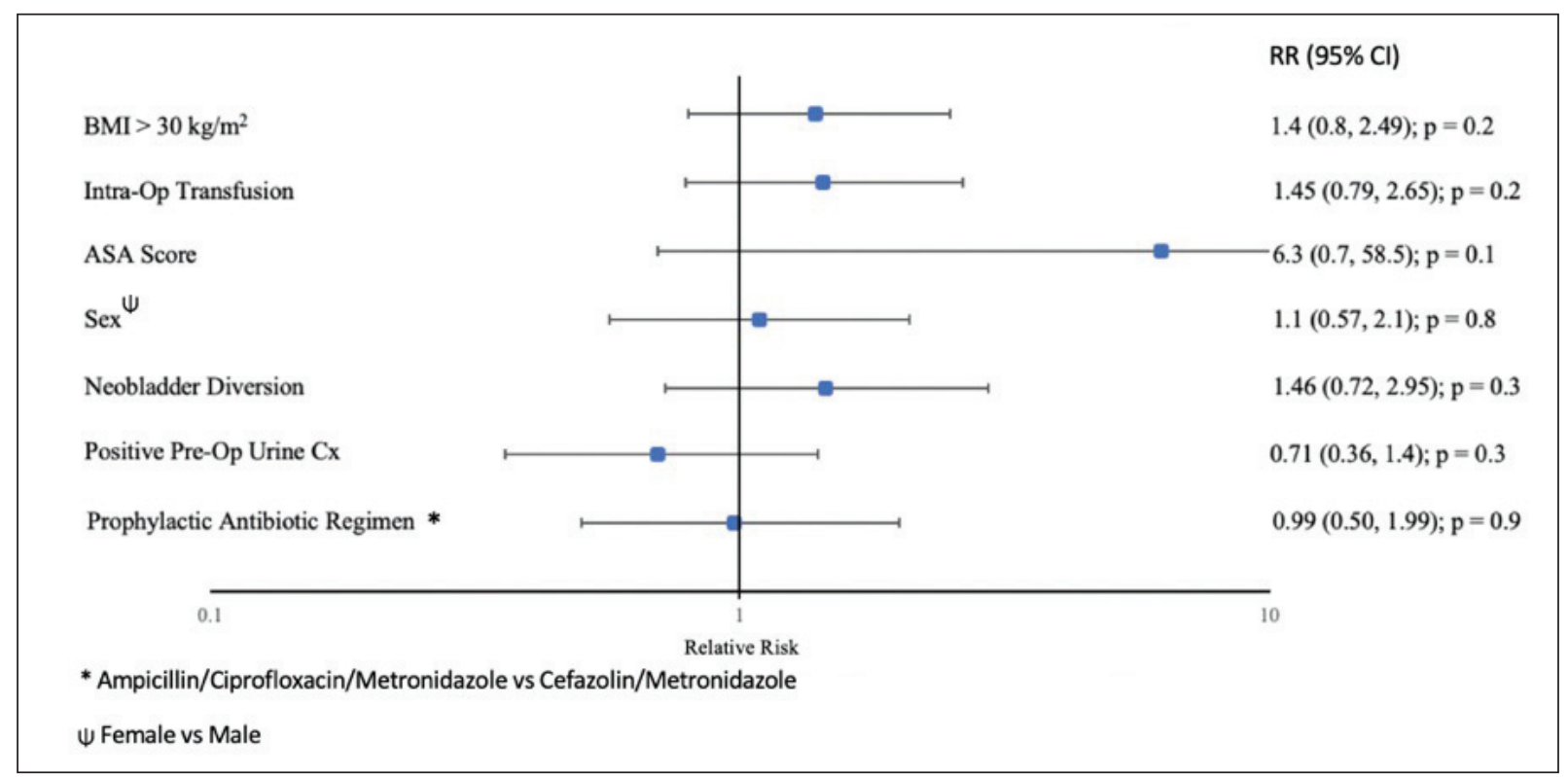

Fig. 3. Multivariable analysis for risk of overall infection post-cystectomy. ASA: American Society of Anesthesiologists; BMI: body mass index; $\mathrm{Cl}$ : confidence interval; RR: relative risk. 


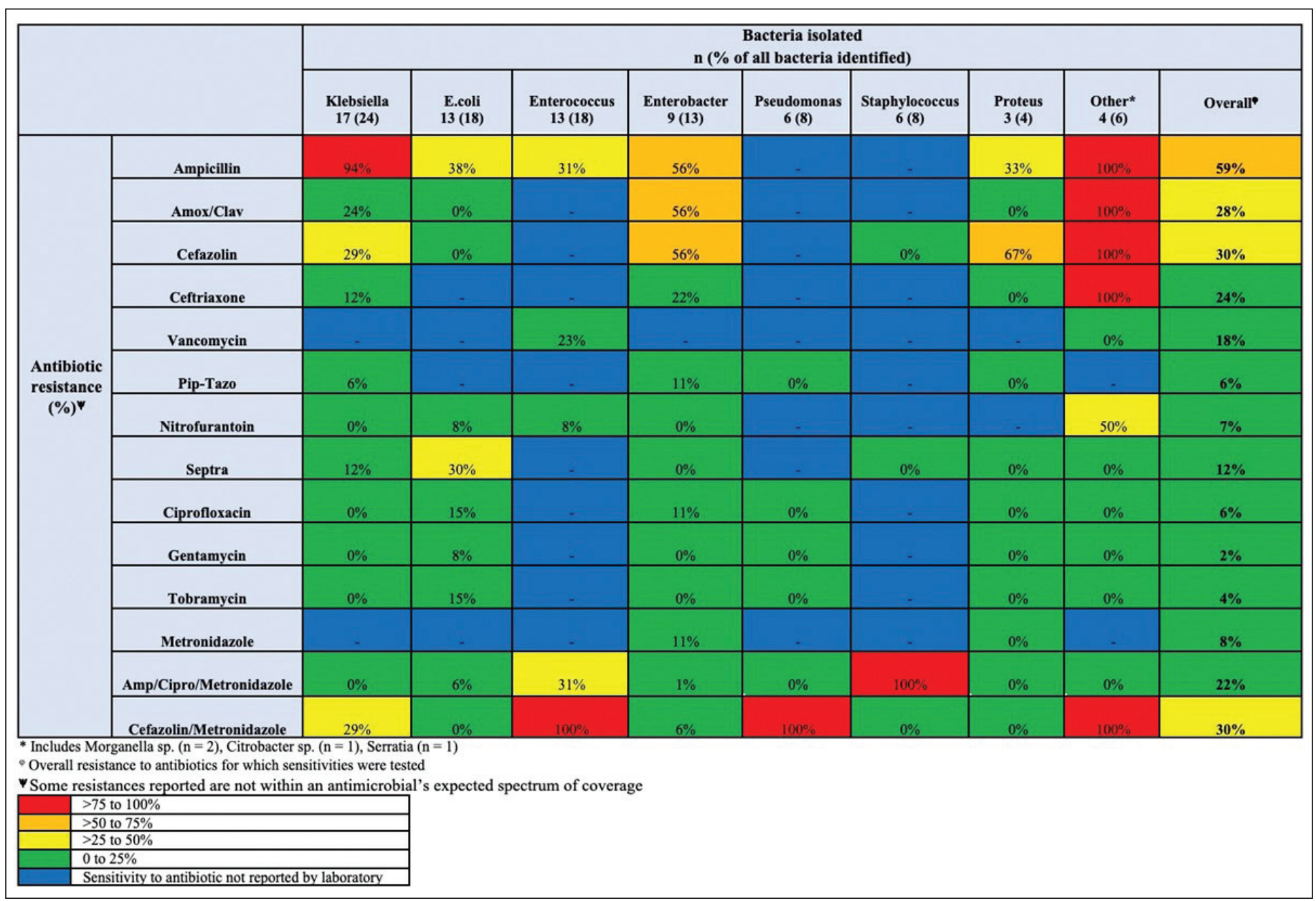

Fig. 4. Proportion of bacteria isolated on postoperative cultures that were resistant to an antimicrobial.

may warrant further study in this population. ${ }^{26}$ Nitrofurantoin is concentrated in the urinary system and is associated with fewer changes in local bacterial flora and less induction of antimicrobial resistance. ${ }^{30}$

The results of this study provide a comprehensive overview of infection post-radical cystectomy at our institution. Because most infections occurred greater than seven days postoperatively, we hypothesize that longer duration of prophylaxis may reduce infections. Furthermore, most bacteria isolated from positive cultures in this study were sensitive to nitrofurantoin and nitrofurantoin is generally considered to have a favorable side effect profile compared to most antimicrobials. Further study is planned to test if routine use of low-dose, daily nitrofurantoin for 2-3 weeks postoperatively is effective and safe.

Limitations of this study include that it was a single-center, retrospective study, therefore generalizability to other centers may not be possible. Lack of randomization may have led to differences between groups. This being said, we adjusted for important patient and treatment variables in the multivariable analysis to reduce confounding. Furthermore, the single physician reviewer was not blinded to prophylactic regimen, representing a potential source of bias. There was no protocol for antimicrobials around time of catheter or stent removal. Because outpatient prescribing data is not routinely recorded in the electronic medical record, we were unable to determine which patients may have received antimicrobials at the time of catheter or stent removal. If differences in postoperative antibiotic use existed between patient groups, this could bias results in a manner that we could not completely control for. However, we attempted to adjust for any possible differences in practice patterns like these by including surgeon as a cluster variable in the multivariable analysis. Some UTIs may have been missed if a urine culture was not drawn or if drawn but negative because antimicrobials had already been initiated. We opted to include only culture-proven UTIs to avoid including patients with symptoms unrelated to infection. Finally, the resistance rates to antimicrobials presented in this study may have been influenced by many factors, including past exposures to hospitals and antimicrobials prior to and at the time of the cystectomy. The resistance patterns reported are not meant to serve as a formal antibiogram. 


\section{Conclusions}

Infection is common after radical cystectomy. This study showed that there was no association between prophylactic antimicrobial regimen and postoperative infection. Based on the results, a further study that includes increasing duration of prophylaxis, as well as the use of more widely sensitive antimicrobials based on local resistance patterns, is being planned to help reduce incidence of infection in this population.

Competing interests: Dr. Hickling has been an advisory board member for Allergan and Pfizer. Dr. Angel has been an advisory board member for Gilead and VilV; has received clinical research grants from Gilead, Merck, and ViiV; and has participated in several clinical trials. Dr. Cagiannos has been an advisory board member for Abbvie, Astellas, and Bayer; and has received grants and/ or honoraria from Abbvie, Astellas, Bayer, and Janssen. Dr. Morash has been an advisory board member for Abbvie, Amgen, Astellas, Ferring, Janssen, Knight, Sanofi, and TerSera. Dr. Lavallée has been an advisory board member for Astellas, Bayer, Ferring, Knight, and Sanofi; and has received an educational grant (unrelated to this work) from Sanofi. The remaining authors do not report any competing personal or financial interests related to this work.

This paper has been peer-reviewed.

\section{References}

1. Parker WP, Tollefson MK, Heins $\mathrm{CN}$, et al. Characterization of perioperative infection risk among patients undergoing radical cystectomy: Results from the national surgical quality improvement program. Urol Oncol Semin Orig Investig 2016;34:532.e13-9. https://doi.org/10.1016/i.urolonc.2016.07.001

2. Krasnow RE, Mossanen M, Koo S, et al. Prophylactic antibiotics and postoperative complications of radical cystectomy: A population-based analysis in the United States. J Urol 2017;198:297-304. https://doi.org/10.1016/i.juro.2017.02.3340

3. Lavallée LI, Schramm D, Witiuk K, et al. Perioperative morbidity associated with radical cystectomy in a multicenter database of community and academic hospitals. PLOS ONE 2014;9:e111281. hitps://doi.org/10.1371//ournal.pone.0111281

4. Haider M, Ladurner C, Mayr R, et al. Use and duration of antibiotic prophylaxis and the rate of urinary tract infection after radical cystectomy for bladder cancer: Results of a multicentric series. Urol Oncol Semin Orig Investig 2019;37:300.e9-15. https://doi.org/10.1016/i.urolonc.2019.01.017

5. Clark JY, Raman JD. Urinary tract infection after radical cystectomy: A vexing problem despite prophylactic antibiotics. Transl Androl Urol 2019;8:S510-3. https://doi.org/10.21037/tau.2019.07.07

6. Kaczmarek K, Lemi ski A, Ba carz A, et al. Postoperative infections among patients undergoing radical cystectomy at a tertiary center. Surg Infect 2018;19:451-8. https://doi.org/10.1089/sur.2017.291

7. Werntz RP, Martinez-Acevedo A, Amadi H. Prophylactic antibiotics following radical cystectomy reduces urinary tract infections and re-admission for sepsis from a urinary source. Urol Oncol Semin Orig Investig 2018;36:238.el-5. https://doi.org/10.1016/j.urolonc.2017.12.025

8. García-Rojo E, Medina-Polo J, Miranda-Utrera N, et al. [Estudio de las infecciones relacionadas con la asistencia sanitaria tras la realización de cistectomía radical.] Actas Urol Esp 2021;45:124-31. https://doi.org/10.1016/j.acuro.2020.06.004

9. Cotter KJ, Fan Y, Sieger GK, et al. Prevalence of clostridium difficile infection in patients after radical cystectomy and neoadjuvant chemotherapy. Bladder Cancer Amst Neth 2017;3:305-10. https://doi.org/10.3233/BLC-170132

10. Mossanen M, Krasnow RE, Lipsitz SR, et al. Associations of specific postoperative complications with costs after radical cystectomy. BJU Int 2018;121:428-36. hitps://doi.org/10.1111/bju.14064

11. Wolters $M$, Oelke $M$, Lutze $B$, et al. Deep surgical site infections after open radical cystectomy and urinary diversion significantly increase hospitalization time and total treatment costs. Urol Int 2017;98:268-73. https://doi.org/10.1159/000449472
12. Davies BJ, Allareddy V and Konety BR. Effect of post-cystectomy infectious complications on cost, length of stay, and mortality. Urology 2009;73:598-602. htrps://doi.org/10.1016/i.urology.2008.09.080

13. Kim SP, Shah ND, Karnes RJ, et al. The implications of hospital acquired adverse events on mortality, length of stay and costs for patients undergoing radical cystectomy for bladder cancer. J Urol 2012;187:2011-7. hitps://doi.org/10.1016/i.juro.2012.01.077

14. Ross J, Breau RH, McAlpine K, et al. A novel prevention bundle to reduce incisional infections after radical cystectomy. Urol Oncol 2020;38:638.e1-6. https://doi.org/10.1016/i.urolonc.2020.04.016

15. Wolf Jr J, Bennet CJ, Dmochowski RR, et al. Urologic Surgery Antimicrobial Prophylaxis - American Urological Association. Modified 2012. Available at: https://www.auanet.org/guidelines/antimicrobial-prophylaxisbest-practice-statement. Accessed July 18, 2019.

16. Pickard R, Bartoletti R, Bierklund-Johansen TE, et al. Guidelines on urological infections - European Association of Urology. Available at: https://uroweb.org/guideline/urologicalinfections/?type=summaryof-changes. Accessed July 18, 2019.

17. Lipp A and Lusardi G. Systemic antimicrobial prophylaxis for percutaneous endoscopic gastrostomy. Cochrane Database Syst Rev 2006:CD005571. https://doi.org/10.1002/14651858.CD005571.pub2

18. Song $F$ and Glenny AM. Antimicrobial prophylaxis in colorectal surgery: A systematic review of randomized controlled trials. Br J Surg 1998;85:1232-41. https://doi.org/10.1046/j.1365-2168.1998.00883.x

19. Anderson DJ, Podgorny K, Berríos-Torres SI, et al. Strategies to prevent surgical site infections in acute care hospitals: 2014 update. Infect Control Hosp Epidemiol Off J Soc Hosp Epidemiol Am 2014;35:605-27. https://doi.org/10.1086/676022

20. Bone RC, Balk RA, Cerra FB, et al. Definitions for sepsis and organ failure and guidelines for the use of innovative therapies in sepsis. The ACCP/SCCM Consensus Conference Committee. American College of Chest Physicians/Society of Critical Care Medicine. Chest 1992;101:1644-55. hitps://doi.org/10.1378/ chest.101.6.1644

21. Pariser JJ, Anderson BB, Pearce SM, et al. The effect of broader, directed antimicrobial prophylaxis including fungal coverage on perioperative infectious complications after radical cystectomy. Urol Oncol Semin Orig Investig 2016;34:121.e9-14. https://doi.org/10.1016/i.urolonc.2015.10.007

22. Clifford TG, Katebian B, Van Horn CM. Urinary tract infections following radical cystectomy and urinary diversion: A review of 1133 patients. World J Urol 2018;36:775-81. https://doi.org/10.1007/ s00345-018-2181-2

23. Mano R, Goldberg H, Stabholz Y, et al. Urinary tract infections after urinary diversion-different occurrence patterns in patients with ileal conduit and orthotopic neobladder. Urology 2018;116:87-92. https://doi.org/10.1016/i.urology.2018.03.042

24. Kolwiick E, Seegers AEM, Tops SCM, et al. Incidence and microbiology of postoperative infections after radical cystectomy and ureteral stent removal: A retrospective cohort study. BMC Infect Dis 2019;19:303. https://doi.org/10.1186/s12879-019-3932-4

25. Hashimoto J, Takahashi S, Kurimura Y, et al. Clinical relevance of single administration of prophylactic antimicrobial agents against febrile events after removal of ureteral stents for patients with urinary diversion or reconstruction. Int J Urol Off J Jpn Urol Assoc 2010;17:163-6. https://doi.org/10.1111/i.14422042.2009.02432.x

26. Kim KH, Yoon HS, Yoon H, et al. Febrile urinary tract infection after radical cystectomy and ileal neobladder in patients with bladder cancer. J Korean Med Sci 2016;31:1100-4. htrps://doi.org/10.3346/ jkms.2016.31.7.1100

27. Abdi H, Elzayat E, Cagiannos I, et al. Female radical cystectomy patients have a higher risk of surgical site infections. Urol Oncol Semin Orig Investig 2018;36:400.el-5. hitps://doi.org/10.1016/i. urolonc.2018.05.023

28. Goldberg H, Shenhar C, Tamir H, et al. Predictors of surgical site infection after radical cystectomy: should we enhance surgical antibiotic prophylaxis? World J Urol 2019;37:1137-43. https://doi.org/10.1007/ s00345-018-2482-5

29. Cek M, Tandoğdu Z, Wagenlehner $F$, et al. Healthcare-associated urinary tract infections in hospitalized urological patients - a global perspective: Results from the GPIU studies 2003-2010. World I Urol 2014;32:1587-94. htrps://doi.org/10.1007/s00345-013-1218-9

30. Squadrito FJ, del Portal D. Nitrofurantoin. In: StatPearls. Treasure Island (FL): StatPearls Publishing 2020. Modified July 13, 2021. Available at: http://www.ncbi.nlm.nih.gov/books/NBK470526/. Accessed Nov. 9, 2020.

Correspondence: Dr. Luke T. Lavallée, Division of Urology, University of Ottawa, Ottawa, ON, Canada; lulavallee@toh.ca 